\title{
Performance of rhesus monkeys on two-cue problems with cues separated*
}

\author{
ISAAC BEHAR \\ U.S. Army Medical Research Laboratory, Fort Knox, Kentucky 40121
}

\begin{abstract}
Relative performance on color and form dimensions during acquisition of two-cue problems by learning-set-sophisticated rhesus monkeys was evaluated with differential reinforcement. Six animals received 24 two-choice problems, each generated from three planometric stimuli: a reference stimulus, an alternate stimulus identical in color but differing in form, and one identical in form but differing in color. The reference stimulus appeared on every trial of a problem and was paired with one or the other alternate on a given trial. On half of the problems, the reference stimulus was positive and on the remainder was negative. Acquisition was nearly equivalent on both cue dimensions with positive reference stimuli, but only one dimension was learned with negative reference stimuli. Results were interpreted as unfavorable to attention theory.
\end{abstract}

The concept of selective attention in recent years has been given a central role in explanations of animal discrimination learning (Mackintosh, 1965; Sutherland, 1964; Sutherland \& Mackintosh, 1971). The historical origins of the contemporary approaches are the noncontinuity positions of Lashley and Wade (1946) and Krechevsky (1938), and in particular that of Lawrence $(1950,1952)$. The essential feature of attentional theories of animal discrimination is that such learning involves two separate processes: learning to attend to the relevant stimulus dimension and learning which responses to attach to specific cue values on the relevant dimension. Because attentional processes are selective, animals are assumed to learn about only one dimension at a time; hence, the more an animal learns about one dimension in a simultaneous two-cue discrimination, the less it learns about the other dimension.

A test of this deduction from attentional theory was made by Sutherland and Holgate (1966) in which rats, initially trained to discriminate between rectangles differing in both brightness and orientation, were given preference tests with stimuli differing only in brightness and only in orientation. Significant negative correlations were obtained between appropriate responses on the two dimensions, as predicted by the two-stage model. A replication of this experiment with cats, by Mumma and Warren (1968), but using transfer tests with differential reinforcement in addition to less sensitive preference tests with nondifferential reinforcement (Warren \& McGonigle, 1969), found no evidence to support the view that the two-cue problems were learned predominantly on the basis of a single dimension.

In both studies the relative amount of learning of the two cues was assessed by tests-preference or

\footnotetext{
* Reprint requests should be sent to Isaac Behar, U.S. Army Aeromedical Research Laboratory, Fort Rucker, Alabama 36360. The author gratefully acknowledges the technical assistance of Bruce A. Bell.
}

transfer-given after the learning criterion was reached on the two-cue discrimination. In view of the contradictory results obtained, a more valid test of whether animals learn two-cue problems on the basis of both cues or predominantly of the more salient cue would be to assess the relative amount learned on the two dimensions during original learning. The present study used a paradigm that avoids the confounding of training on the two dimensions. It consisted of two-choice simultaneous discriminations comprising three stimuli: a reference stimulus that appeared on all trials and two alternate stimuli, each of which appeared on half of the trials. One alternate stimulus differed from the reference stimulus in color but was identical in form, while the other alternate stimulus differed from the reference stimulus in form but was identical in color. Thus, over a series of trials, the animal was confronted with both a form and a color discrimination, with a stimulus in common in the two discriminations. If the animal attended to or otherwise utilized the information about both cue dimensions, learning on both discriminations would be expected to progress at the same rate. Whereas, if he responded only in terms of the more salient cue dimension, learning on the discrimination based on the less salient cue dimension would remain at chance level.

Another point of interest in this study was the difference in performance that might be obtained if the reference stimulus was the positive stimulus and the other two stimuli were negative or, alternatively, if the reference stimulus was the negative stimulus and the other two positive. In addition to performance in acquisition, differential resistance to extinction was also examined as a measure of preference for the two cue dimensions.

\section{METHOD}

Subjects

Six young adult male rhesus monkeys (Macaca mulatta) served 


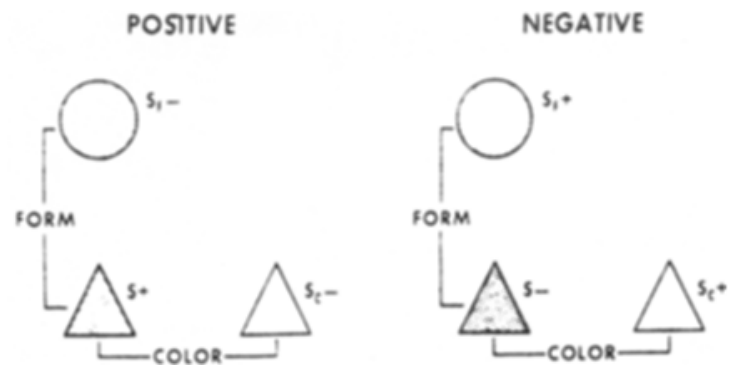

Fig. 1. Schematization of the experimental paradigms. The reference stimulus in the positive problems is designated $\mathrm{S}+$. while in the negative problems it is $\mathbf{S}$-.

as Ss. All animals wcre feral. Prior to the present study, they had participated in one experiment, in which they had been trained in a 400-problem object-quality learning set.

\section{Apparatus}

The Emory version (Riopelle. 1954) of the WGTA was modified so that the raising and lowering of the opaque screen (which separates the animal from the test tray) was controlled by a footswitch-operated motor-driven lead screw and nut. Timing switches, suitably located, permitted the measurement of response latency. Stimuli consisted of 72 planometric objects that were combined to form 24 problems. The stimuli were regular geometric figures and colors were homogeneous. There were 32 unique colors and 25 unique forms, with random assignment to problems.

\section{Procedure}

Each animal received 24 problems, one each day. Each problem consisted of an intermixed series of trials on both a form and color discrimination. A trial consisted of the presentation of a reference and an alternate stimulus for a simultaneous two-choice visual discrimination. The reference stimuli, which appeared on every trial of a problem, were consistently rewarded $(\mathbf{S}+)$ on half of the problems (positive problems) and were consistently nonrewarded $(S-)$ on the remaining problems (negative problems). In positive problems, both alternate stimuli were nonrewarded $\left(\mathrm{S}_{\mathrm{f}^{-}}, \mathrm{S}_{\mathrm{c}^{-}}\right)$; in negative problems, both alternate stimuli were rewarded $\left(\mathrm{S}_{\mathrm{f}^{+}}, \mathrm{S}_{\mathrm{c}^{+}}\right)$. These paradigms are depicted in $\mathrm{Fig}$. 1. Positive and negative problems were alternated on successive days. For three animals the first problem received was a positive problem, for the others it was negative.

The choice of alternate stimulus on any trial was randomly determined, with the constraint that each four-trial block contained two trials with each of the alternate stimuli. The acquisition phase consisted of training on a problem until the criterion of 9 correct responses on 10 consecutive trials was met on either the form or color dimension. Any remaining trials within the four-trial block in which criterion was met were also completed. The spatial position of the correct stimulus was determined by Gellermann orders. Following acquisition of each problem, without any change in the intertrial interval, extinction trials with the alternate stimuli were given. For the positive problems, this consisted of pairing the two negative stimuli, while, for the negative problems, the positive stimuli were paired. The extinction criterion was met when the animal failed to respond to either stimulus within $20 \mathrm{sec}$ on three consecutive trials.

The specific procedure on a given trial consisted of the following. With the opaque screen lowered between the animal and the test tray, the foodwell to be covered by the positive stimulus was baited with a reward (raisin. peanut, or apple section). Both stimuli were then placed over the foodwells and the one-way screen was lowered. Next, the opaque screen was raised. permitting response. The noncorrection method was used throughout. On extinction trials neither foodwell was baited.

\section{RESULTS}

\section{Acquisition}

The mean number of trials required to attain the acquisition criterion (including the criterional trials) was 12.9 and 12.0 , respectively, for the positive and negative problems. The criterion was met in somewhat fewer trials in the last block of three problems (11.1) than in the first block (13.9), but the differences among blocks, between positive and negative problems, and in the interaction between these variables were all not statistically significant.

The dimension for which criterion was attained for the positive problems was color on $38.9 \%$ of the problems, form on $36.1 \%$, and both dimensions on $25 \%$ of the problems. For the negative problems color was the salient dimension on $51.4 \%$ of the problems, form on $41.7 \%$, and both equally on $6.9 \%$. The difference in the number of problems during which the criterion was attained simultaneously on both dimensions is significantly higher for the positive than for the negative problems $(t=3.61, p<.02)$. Differences in the relative frequency of color and form saliency are not significant for either the positive or negative problems.

An estimate of the amount learned on the less salient dimension is provided by the percentage of correct responses during the 10 trials on that dimension which were presented at a corresponding time, that is, intermixed with the 10 trials that constituted criterion attainment for the learned dimension. For the positive problems the percentage correct on the higher dimension was $91.9 \%$, while for the lower dimension it was $77.9 \%$. For the negative problems these percentages were $93.6 \%$ and $57.9 \%$. The differences between the positive and negative problems, the higher and lower dimensions, and the interaction between these variables are all significant at the .005 level or better. The percent correct responses on the lower dimension $(77.9 \%)$ on the positive problems is significantly greater than chance, while that for the negative problems $(57.9 \%)$ does not differ reliably from chance. The slightly greater percentage correct on the learned dimension for the negative (93.6\%) than for the positive $(91.9 \%)$ problems is also statistically significant $(F=7.5, \mathrm{df}=1 / 5, \mathrm{p}<.05)$.

For a more specific test of the Sutherland and Holgate assumption of an inverse relation between the performance on the two dimensions, the product-moment correlation coefficient was calculated between the number of errors made on the color and form dimensions. For the first positive problem presented, the obtained $r$ was 0.898 , which for $\mathrm{N}=6$ is 
significant at the .01 level. For the first negative problem, the corresponding $\mathrm{r}$ was -0.751 , which has a $\mathrm{p}$ value of less than .05 . The correlation coefficient was also determined for each animal separately based on the 12 positive and 12 negative problems that each received. For the positive problems, these ranged from .280 to 973 . with a mean $\mathrm{r}$ of 0.728 . Using the Fisher method for combining significance levels, the combined $p$ is less than .00001 . For the negative problems, the is for individual animals ranged from .073 to -.523 , with a mean $\mathrm{r}$ of -0.212 . For a two-tailed test the combined probabilities are not significant but, if one allows a one-tailed test, significance is obtained with $p=.0375$.

Since learning was very rapid, an analysis was performed to determine whether the dimension learned was the one on which the first correct (reinforced) response was made. Excluding the problems in which the color and form discriminations reached criterion at the same time, the percentage of problems in which the dimension of the first correct responses and that learned were the same was $42.6 \%$ for the positive problems (not significantly different from chance) but was $71.6 \%$ for the negative problems (significant at the .025 level, $\mathrm{F}=15.0, \mathrm{df}=1 / 5)$.

\section{Choice Responses}

Responses on the first extinction trial of each problem were analyzed separately, as they constitute a choice or preference test between the alternate stimuli, which during acquisition never appeared together. For the positive problems, excluding those in which criterion was attained on both dimensions, the alternate negative stimuli of the learned dimension were chosen more frequently than were the negative stimuli of the less learned dimension ( $53.7 \%$ vs $46.3 \%$ ), but this difference was not significant. The corresponding percentages for the negative problems were $85.1 \%$ to the alternate positive of the learned dimension and $14.9 \%$ to that of the less learned dimension, a highly signific ant difference $(\mathrm{F}=169.8, \quad \mathrm{df}=1 / 5, \mathrm{p}<.001)$. On the positive problems the percentage choice of the alternate stimuli differed as a function of which dimension was learned to criterion (see Fig. 2). When form was the learned dimension, the animals chose the negative alternates of the same dimension in $69.2 \%$ of the problems, while, when color was the learned dimension, the negative alternates of the other dimension were more frequently chosen (60.7\%). Thus, in both cases, the animals more frequently chose the negative alternate stimuli that were identical to the reference positive stimuli in color, regardless of whether color or form was the learned dimension. The interaction upon which this analysis is based is statistically significant $(\mathrm{F}=10.0, \mathrm{df}=1 / 5$, $\mathrm{p}<.05)$. No corresponding effect occurred for the negative problems.

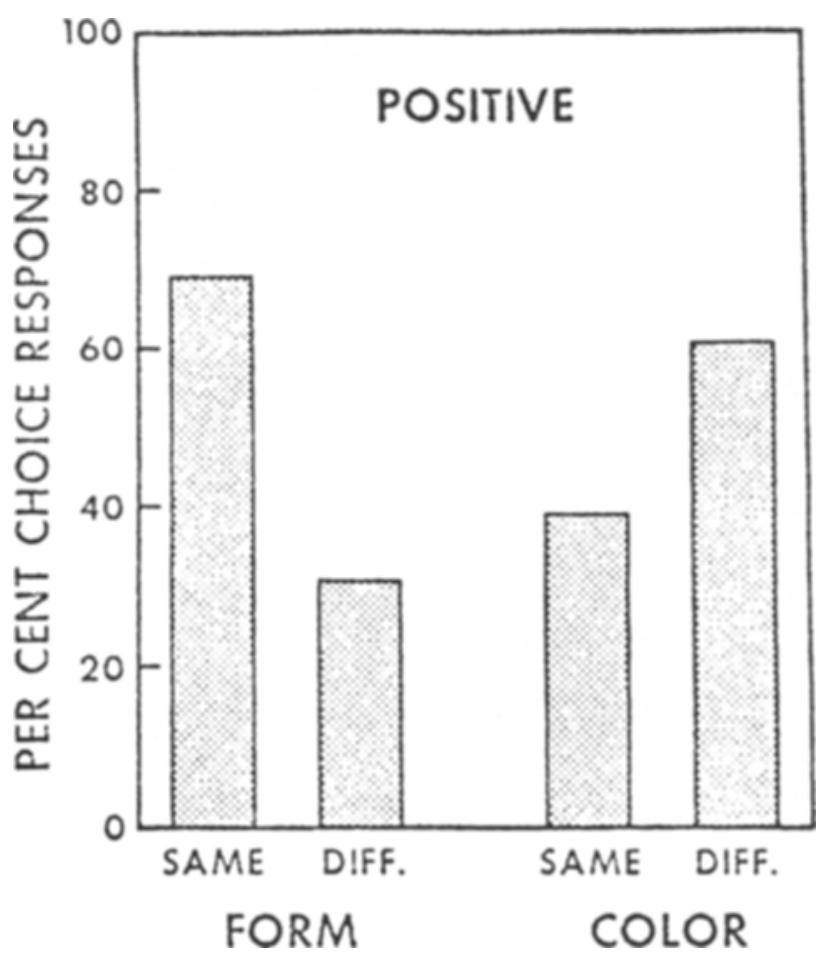

Fig. 2. The percent choice responses of the negative stimuli that were from the same and different dimension as that learned when the learned dimension was form and color.

\section{Extinction}

The mean number of extinction responses made on the positive and negative problems varied over blocks of problems, as depicted in Fig. 3. The decline in the number of responses made before reaching the extinction criterion was significant $(\mathrm{F}=8.5, \mathrm{df}=3 / 15$, $\mathrm{p}<.005$ ); however, the interaction of Blocks by Type of Problem was not significant.

The mean number of extinction responses made to the alternate negative stimuli on the positive problems was slightly lower for the alternate of the same dimension (4.35) as that learned than for the other dimension (5.00), while for the negative problems the positive alternate of the same dimension was responded to nearly twice as frequently $(6.12)$ as were those of the other dimension (3.27).

Paralleling the analysis of choice responses, an analysis was made of the frequency of responding during extinction to the alternate stimuli as a function of the learned dimension. For the positive problems, the number of responses to the alternate stimuli are depicted in Fig. 4. The difference in the mean number of extinction responses when the learned dimension was form and color was not significant. The interaction between the learned dimension and the dimension of the alternate stimuli (i.e., same or different) was significant $(\mathrm{F}=8.7, \mathrm{df}=1 / 5, \mathrm{p}<.05)$ : however, this effect is based 


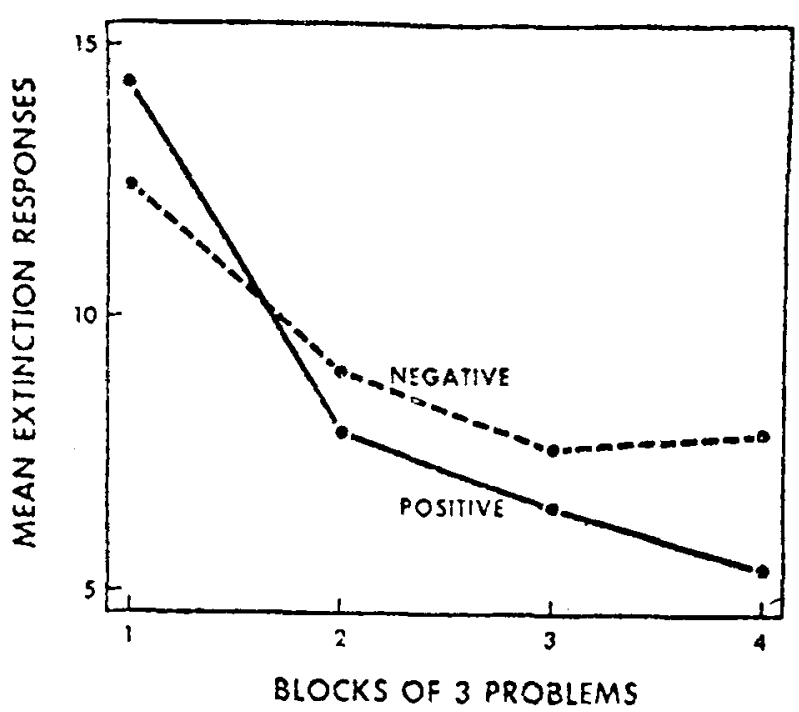

Fig. 3. Resistance to extinction as a function of problem blocks for the negative and positive problems.

entirely upon the difference in the mean number of responses to the two alternate stimuli when color was the learned dimension. Here the resistance to extinction was significantly greater $(F=21.7, \mathrm{df}=1 / 5, \mathrm{p}<.01)$ for the alternates identical in color to the reference stimuli (6.58 mean responses) than for the alternates identical in form (5.37 mean responses).

\section{DISCUSSION}

The performance on the positive problems provides no support for the two-stage theory. Learning on both dimensions was nearly comparable and correlations between the two dimensions were positive instead of negative and relatively high. Although these results appear damaging, it must be recalled that the animals in the study were learning-set-sophisticated monkeys. Perhaps, during learning-set formation with multidimensional ("junk") stimuli, monkeys learn to attend to stimulus differences in a number of dimensions (that is, they learn to scan a number of different analyzers), so that learning to switch in the correct analyzer could occur in a single trial. Indeed, the one-trial learning of learning-set-sophisticated monkeys may be based principally on the very astute observing behavior resulting from this experience. A test of this proposition can be made by comparing learning-set performance of monkeys given an equivalent amount of experience on either a series of conventional multidimensional problems or unidimensional problems. If the interpretation of the change in observing behavior with learning-set acquisition is correct, then one would expect less evidence of cue dominance in such monkeys. In relatively naive monkeys, color is so overpowering a cile that discriminations differing only in color are learned as rapidly as those differing in both color and form (Harlow, 1945) or in color, form, and size (Warren, 1953). In the present study, color was not significantly more salient a cue dimension than was form during acquisition, although slight evidence for its greater salience occurred in the preference tests. Thus, it appears that (perhaps innate) cue dominance hierarchies are neutralized by learning-set formation.

If we discount the apparently damaging evidence against the two-factor theory provided by the results of the positive problems, we could not with logical consistency offer the results of the negative problems as favorable evidence, much as these results appear supportive. How, then, to account for the chance performance on the less learned cue dimension and the negative correlations predominantly obtained?

First of all. let us lay to rest an explanation based upon responses to the familiar or novel cue per se. In the positive problems the positive stimulus was present on all trials and, hence, was the more familiar cue. If the animals learned to respond to familiarity per se, no differential dimensional salience would have been expected, and the results would be well accounted for. However, in the negative problems, if familiarity was the basis for response, performance on both dimensions should have been nondifferentially impaired but was not. Interpretation based upon novelty suffers a similar limitation.

The pattern of results for both the positive and negative problems suggests that, while attention to both

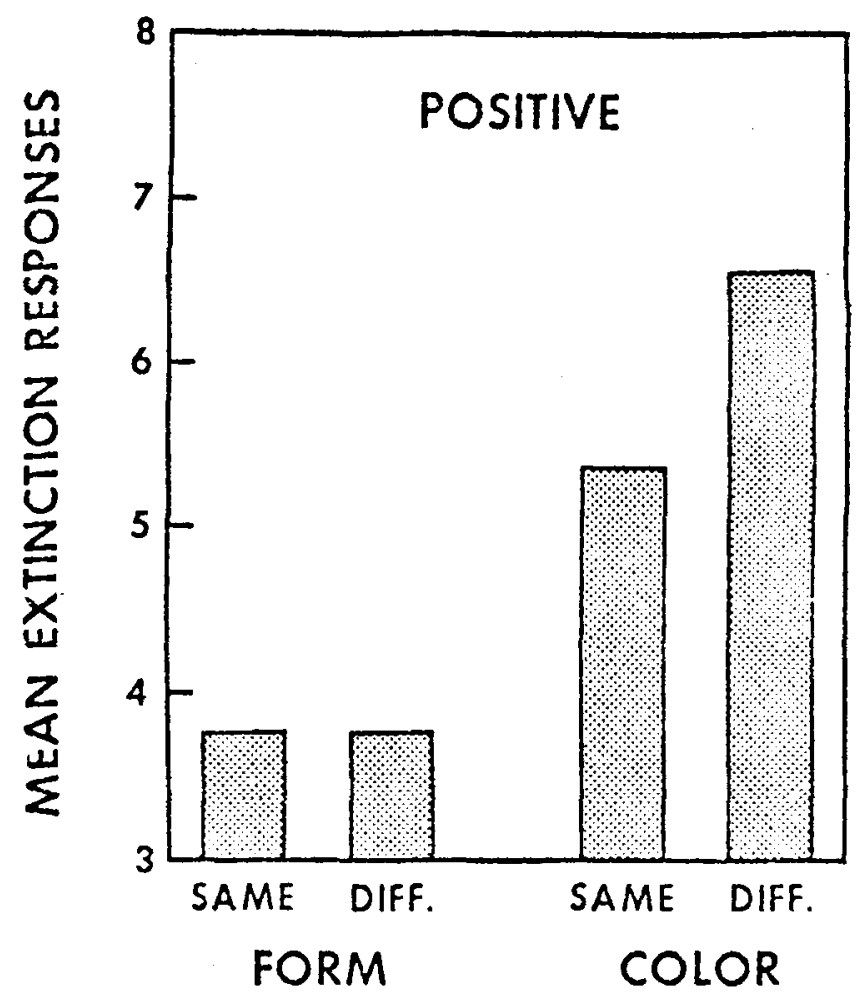

Fig. 4. The mean extinction responses to the negative stimuli that were from the same and different dimension as that learned when the learned dimension was form and color. 
cue dimensions may be equivalent, the functional role of the positive and negative cues is decidedly noncomplementary. That is, the associative processes linking responses to cue values that signal reward and to those that signal nonreward are not equivalent. Indeed, learning which cue values signal reward is virtually all that is learned: very little aversiveness (often the opposite) accrues to the negative stimulus (see also Leary, 1956: Behar, 1962). From this argument, given the very rapid learning that occurred and assuming that both color and form analyzers are at asymptote, the animal first identifies the cue value that signals reward. On trials in which that cue value is absent, in the negative problems, the animal is confronted with a negative, but not aversive, stimulus that is identical to the identified positive stimulus in one of the two dimensions and a positive stimulus that resembles it not at all. Clearly, learning on this dimension is impaired by the generalization that occurs from the positive cue of the first learned dimension.

\section{REFERENCES}

Behar, 1. Evaluation of the significance of positive and negative cue in discrimination learning. Journal of Comparative \& Physiological Psychology, 1962, 55, 502-504.

Harlow, H. F. Studies in discrimination learning by monkeys: VI. Discriminations between stimuli differing in both color and form, only in color, and only in form. Journal of General Psychology, 1945, 33, 225-235.

Krechevsky, I. A study of the continuity of the problem-solving process. Psychological Review, 1938, 45, 107-133.

Lashley, K. S., \& Wade, M. The Pavlovian theory of generalization. Psychological Review, 1946, 53, 72-87.

Lawrence, D. HI. Acquired distinctiveness of cues: I. Transfer between discriminations on the basis of familiarity with the stimulus. Journal of Experimental Psychology, 1949, 39, 770-784.

Lawrence, D. H. Acquired distinctiveness of cues: II. Selective association in a constant stimulus situation. Journal of Experimental Psychology, 1950, 40, 175-188.

Leary, R. W. The rewarded, the unrewarded, the chosen, and the unchosen. Psychological Reports, 1956, 2, 91-97.

Mackintosh, N. J. Selective attention in animal discrimination learning. Psychological Bulletin, 1965, 64, 124-150.

Mumma, R., \& Warren, J. M. Two-cue discrimination learning by cats. Journal of Comparative \& Physiological Psychology, 1968, 66, 116-121

Riopelle, A. J. The facilities of the Emory University primate behavior laboratory. Journal of Psychology, 1954, 38, 331-338

Sutherland, N. S. The learning of discrimination by animals. Endeavour, 1964, 23, 148-152.

Sutherland, N. S., \& Holgate, V. Two-cue discrimination learning in rats. Journal of Comparative \& Physiological Psychology, $1966,61,198-207$

Sutherland, N. S., \& Mackintosh, N. J. Mechanisms of animal discrimination learning. New York: Academic Press, 1971.

Warren, J. M. Additivity of cues in visual pattern discriminations by monkeys. Journal of Comparative \& Physiological Psychology, 1953, 46, 484-486.

Warren, J. M., \& McGonigle, B. Attention theory and discrimination learning. In R. Gilbert and N. S. Sutherland (Eds.), Animal discrimination learning. London: Academic Press, 1969. Pp. 113-136.

(Received for publication June 11, 1973;

revision received September 4, 1973; accepted September 13,1973.) 